\title{
Background Subtraction using Adaptive Singular Value Decomposition
}

\author{
Günther Reitberger ${ }^{1} \mathbb{D} \cdot$ Tomas Sauer $^{1}$
}

Received: 4 July 2019 / Accepted: 15 May 2020 / Published online: 5 June 2020

(C) The Author(s) 2020

\begin{abstract}
An important task when processing sensor data is to distinguish relevant from irrelevant data. This paper describes a method for an iterative singular value decomposition that maintains a model of the background via singular vectors spanning a subspace of the image space, thus providing a way to determine the amount of new information contained in an incoming frame. We update the singular vectors spanning the background space in a computationally efficient manner and provide the ability to perform blockwise updates, leading to a fast and robust adaptive SVD computation. The effects of those two properties and the success of the overall method to perform a state-of-the-art background subtraction are shown in both qualitative and quantitative evaluations.
\end{abstract}

Keywords Image processing · Background subtraction · Singular value decomposition

\section{Introduction}

With static cameras, for example in video surveillance, the background, like houses or trees, stays mostly constant over a series of frames, whereas the foreground consisting of objects of interest, e.g., cars or humans, causes differences in image sequences. Background subtraction aims to distinguish between foreground and background based on previous image sequences and eliminates the background from newly incoming frames, leaving only the moving objects contained in the foreground. These are usually the objects of interest in surveillance.

\subsection{Motivation}

Data-driven approaches are a major topic in image processing and computer vision, leading to state-of-the-art performances, for example in classification or regression tasks. One example is video surveillance used for security reasons, traffic regulation, or as information source in autonomous driving. The main problems with data-driven approaches are that the training data have to be well balanced and to cover all scenarios that appear later in the

Günther Reitberger

reitberg@forwiss.uni-passau.de

Tomas Sauer

sauer@forwiss.uni-passau.de

1 FORWISS, University of Passau, Passau, Germany execution phase and have to be well annotated. In contrast to cameras mounted at moving objects such as vehicles, static cameras mounted at some infrastructure observe a scenery, e.g., houses, trees, parked cars, that is widely fixed or at least remains static over a large number of frames. If one is interested in moving objects, as it is the case in the aforementioned applications, the relevant data are exactly the one different from the static data. The reduction of the input data, i.e., the frames taken from the static cameras, to the relevant data, i.e., the moving objects, is important for several applications like the generation of training data for machine learning approaches or as input for classification tasks reducing false positive detections due to the removal of the irrelevant static part.

Calling the static part background and the moving objects foreground, the task of dynamic and static part distinction is known as foreground background separation or simply background subtraction.

\subsection{Background Subtraction as Optimization Problem}

Throughout the paper, we make the assumptions that the camera is static, the background is mostly constant up to rare changes and illumination, and the moving objects, considered as foreground, are small relative to the image size. Then, background subtraction can be formulated as an optimization problem. Given an image sequence stacked in 
vectorized form into the matrix $A \in \mathbb{R}^{d \times n}$, with $d$ being the number of pixels of an image and $n$ being the number of images, foreground-background separation can be modeled as decomposing $A$ into a low-rank matrix $L$, the background and a sparse matrix $S$, the foreground, cf. [8]. This leads to the optimization problem

$\min _{L, S} \operatorname{rank}(L)+\lambda\|S\|_{0} \quad$ s.t. $\quad A=L+S$.

Unfortunately, solving this problem is not feasible. Therefore, adaptations have to be made. Recall that a singular value decomposition (SVD) decomposes a matrix $A \in \mathbb{R}^{d \times n}$ into

$A=U \Sigma V^{T}$

with orthogonal matrices $U \in \mathbb{R}^{d \times d}$ and $V \in \mathbb{R}^{n \times n}$ and the diagonal matrix

$\Sigma=\left[\begin{array}{rr}\Sigma^{\prime} & 0 \\ 0 & 0\end{array}\right] \in \mathbb{R}^{d \times n}, \quad \Sigma^{\prime} \in \mathbb{R}^{r \times r}, \quad r=\operatorname{rank} A$,

where $\Sigma^{\prime}$ has strictly positive diagonal values. The SVD makes no relaxation of the rank, but, given $\ell \leq r$, the best (in an $\ell_{2}$ sense) rank- $\ell, \ell \in \mathbb{N}$, estimate $L$ of $A$ can be obtained by using the first $\ell$ singular values and vectors, see $[28,29]$. This solves the optimization problems

$\min \|A-L\|_{F} \quad$ or $\|A-L\|_{2} \quad$ s.t. $\quad \operatorname{rank} L \leq \ell$.

We use the following notation throughout our paper: $U_{:, 1: \ell}:=$ $U(:, 1: \ell):=\left[u_{1}, \ldots, u_{\ell}\right]$, with $u_{i}$ being the $i$ th column of $\mathrm{U}, i \in\{1, \ldots, \ell\}$.

The first $\ell$ columns of the $U$ matrix of the SVD (2) of $A$, i.e., the left singular vectors corresponding to the $\ell$ biggest singular values, span a subspace of the column space of $A$. The background of an image $J \in \mathbb{R}^{d \times 1}$ is calculated by the orthogonal projection of $J$ on $U_{\ell}:=U_{:, 1: \ell}$ by $U_{\ell}\left(U_{\ell}^{T} J\right)$. The foreground then consists of the difference in the background from the image $J-U_{\ell}\left(U_{\ell}^{T} J\right)=\left(I-U_{\ell} U_{\ell}^{T}\right) J$.

The aim of a surveillance application is to subtract the background from every incoming image. Modeling the background via (3) results in a batch algorithm, where the low-rank approximations are calculated based on some (recent) sample frames stacked together to the matrix $A$. Note that this allows the background to change slowly over time, for example due to changing illumination or to parked cars leaving the scene. It is well known that the computational effort to determine the SVD of $A$ with dimensions $d \gg n$ is $O\left(d n^{2}\right)$ using R-SVD and computing only $U_{n}=U_{:, 1: n}$ instead of the complete $d \times d$ matrix $U$, and the memory consumption is $O(d n)$, cf. [10]. Especially in the case of higher definition images, only rather few samples $n$ can be used in this way. This results in a dependency of the background model from the sample image size and an inability of adaption to a change in the background that is not covered in the few sample frames. Hence, a naive batch algorithm is not a suitable solution.

\subsection{Main Contributions and Outline}

The layout of this paper is as follows. In Sect. 2, we briefly revise related work in background subtraction and SVD methods. Section 3 introduces our algorithm of iteratively calculating an SVD. The main contribution here consists in the application and adaption of the iterative SVD to background subtraction. In Sect. 4, we propose a concrete algorithm that adapts the model of the background in a way that is dependent on the incoming data because of which we call it adaptive SVD. A straightforward version of the algorithm still has limitations, because of which we present extensions of the basic algorithm that overcome these deficits. In Sect. 5, evaluations of the method give an impression of execution time, generality, and performance capabilities of the adaptive SVD. Finally, in Sect. 6 our main conclusions are outlined.

\section{Related Work}

The "philosophical" goal of background modeling is to acquire a background image that does not include any moving objects. In realistic environments, the background may also change, due to influences like illumination or objects being introduced to or removed from the scene. Considering these problems as well as robustness and adaptation, background modeling methods can, according to the survey papers $[2,3,6]$, be classified into the following categories: statistical background modeling, background modeling via clustering, background estimation, and neural networks.

The most recent approach is, of course, to model the background via neural networks. Particularly, convolutional neural networks (CNNs) [15] have performed very well in many tasks of image processing. These techniques, however, usually involve a labeling of the data, i.e., the background has to be annotated, mostly manually, for a set of training images. The network then learns the background based on the labels. Gracewell and John [12], for example, use an autoencoder network architecture for the training of a background model based on labeled data. Background modeling is often combined with classification or segmentation tasks where every pixel of an image is assigned to one class. Based on the classes, the pixel can then be classified as background or foreground, respectively. This task is often done by means of transfer learning, cf. [11, p. 526]. Following this idea, Lim and Keles [17] add three layers to a pre- 
trained image content classifying $\mathrm{CNN}$ and post-train their resulting network on few labeled foreground segmentations. Such techniques strongly depend on the training data and can only be improved by adding further data. An evaluation of the network performance depending on the training data is made in [20], and an overview of neural networks for background subtraction is provided in [4]. Reinforcement learning [21] and unsupervised learning, on the other hand, do not need labeled data for training. Sultana et al. [30] extend a pre-trained $\mathrm{CNN}$ with an unsupervised network to generate background at the image positions where objects are detected. Therefore, their approach does not require labeled data, at least not for the post-training. Our algorithm, on the other hand, is data driven as well, but it is flexible and does not have to be fully re-trained if the application data are essentially different from the training data; this feature strongly contrasts with the aforementioned neural network approaches.

Statistical background modeling includes Gaussian models, support vector machines, and subspace learning models. Subspace learning originates from the modeling of the background subtraction task as shown in (1). Our approach therefore also belongs to this domain. Principal component pursuit (PCP) [8] is based on the convex relaxation of (1) by

$\min _{L, S}\|L\|_{*}+\lambda\|S\|_{1} \quad$ s.t. $\quad A=L+S$,

with $\|L\|_{*}$ being the nuclear norm of matrix $L$, the sum of the singular values of $L$. Relaxation (4) can be solved by efficient algorithms such as alternating optimization. As PCP considers the $\ell_{1}$ error, it is more robust against outliers or salt-and-pepper noise than SVD-based methods and thus more suited to situations that suffer of that type of noise. Since outliers are not a substantial problem in traffic surveillance, which is our main application in mind, we do not have to dwell on this type of robustness. In addition, the pure PCP method also has its limitations such as being a batch algorithm, being computationally expensive compared to SVD, and maintaining the exact rank of the low-rank approximation, cf. [6]. This is a problem when it comes to data that are affected by noise in most components, which is usually the case in camera-based image processing. We remark that to overcome the drawbacks of plain PCP, many extensions of the PCP have been developed. Rodriguez and Wohlberg introduce an incremental PCP algorithm in [24] and extend it in [25] by an optimization step to cope with translational and rotational jitter. Incremental PCP is able to adapt to a gradually changing low-rank subspace, which is the case with video data in the field of background subtraction.

Generally, the approach to solve (1) by some relaxation, like PCP does, is also called robust principal component anal- ysis (RPCA). Static approaches assume a constant low-rank subspace, whereas dynamic ones incorporate a gradually changing low-rank subspace. Recursive projected compressive sensing (ReProCS) described by Guo et al. [13] and the ReProCS-based algorithm MERoP described in [22] are examples for a solution of the dynamic RPCA problem, just like [24] mentioned above. An overview of RPCA methods is given in [5,31].

As already mentioned, we want to focus on $\ell_{2}$ regularization and therefore SVD-based methods. In recent years, several algorithms have been developed to speed up the calculation of the SVD, for finding the optimal rank- $\ell$ matrix approximating a given data matrix that is usually high dimensional and dense, i.e., of high rank, in the domain of background subtraction. Liu et al. [18] iteratively approximate the subspace of dimension $\ell$ via a block Krylov subspace optimization approach. Although this is fast, convergence assumptions have to be met. Another popular way for a fast SVD calculation is by randomization. Erichson et al. use random test matrices in [9] followed by a compressed sensing technique to approximate the dominant left and right singular vectors. Kaloorazi and de Lamare offer in [14] a decomposition of the data matrix into $U Z V$ with $Z$ allowing only small off-diagonal entries in an $\ell_{2}$ sense. This factorization is rank revealing and can be efficiently calculated via random sampling. To further speed up the randomized approaches, which tend to have good parallelization abilities, Lu et al. offer a way to blockwisely move parts of the calculations onto the GPU in [19]. For our application, however, it is important that the low-rank subspace calculation algorithm is extendable to an iteratively growing data matrix which is not available in the aforementioned fast SVD calculation approaches.

There is naturally a close relationship between our SVDbased approach and incremental principal component analysis (PCA) due to the close relationship between SVD and PCA. Given a matrix $A \in \mathbb{R}^{n \times d}$ with $n$ being the number of samples and $d$ the number of features, the PCA searches for the first $k$ eigenvectors of the correlation matrix $A^{T} A$ which span the same subspace as the first $k$ columns of the $U$ matrix of the SVD of $A^{T}$, i.e., the left singular vectors of $A^{T}$. Thus, usually the PCA is actually calculated by an SVD; since $A^{T}=U \Sigma V^{T}$ gives $A^{T} A=$ $U \Sigma V^{T} V \Sigma U^{T}=U \Sigma^{2} U^{T}$, the PCA produces the same subspace as our iterative SVD approach. One difference is that PCA originates from the statistics domain and the applications search for the main directions in which the data differ from the mean data sample. That is why, the matrix $A$ usually gets normalized by subtraction of the columnwise mean and divided by the columnwise standard deviation before calculating the PCA which, however, makes no sense in our application. This is also expressed in the work by 
Ross et al. [26], based on the sequential Karhunen-Loeve basis extraction from [16]. They use the PCA as a feature extractor for a tracking application. In our approach, we model the mean data, the background, by singular vectors only and dig deeper into the application to background subtraction, which has not been considered in the PCA context. Nevertheless, we will make further comparisons to PCA, pointing out similarities and differences to our approach.

\section{Update Methods for Rank Revealing Decompositions and Applications}

Our background subtraction method is based on an iterative calculation of an SVD for matrices augmented by columns, cf. [23]. In this section, we revise the essential statements and the advantages of using this method for calculating the SVD.

\subsection{Iterative SVD}

The method from [23] is outlined, in its basic form, as follows:

- Given: SVD of $\mathbb{R}^{d \times n_{k}} \ni A_{k}=U_{k} \Sigma_{k} V_{k}^{T}, n_{k} \ll d$, and $\operatorname{rank}\left(A_{k}\right)=: r_{k}$

- Aim: Compute SVD for $A_{k+1}=\left[A_{k}, B_{k}\right]$,

$B_{k} \in \mathbb{R}^{d \times m_{k}}, m_{k}:=n_{k+1}-n_{k}$

- Update: $A_{k+1}=U_{k+1} \Sigma_{k+1} V_{k+1}^{T}$ with

$$
\begin{aligned}
U_{k+1} & =U_{k} Q\left[\begin{array}{ll}
\tilde{U} & 0 \\
0 & I
\end{array}\right], \\
V_{k+1} & =\left[\begin{array}{ll}
V_{k} & 0 \\
0 & I
\end{array}\right]\left(P_{k}^{\prime} P_{k}\right)^{T}\left[\begin{array}{cc}
\tilde{V} & 0 \\
0 & I
\end{array}\right],
\end{aligned}
$$

where $Q$ results from a QR-decomposition, $\Sigma_{k+1}, \tilde{U}$ and $\tilde{V}$ result from the SVD of a $\left(r_{k}+m_{k}\right) \times\left(r_{k}+m_{k}\right)$ matrix. $P_{k}$ and $P_{k}^{\prime}$ are permutation matrices.

For details, see [23]. In the original version of the iterative $\mathrm{SVD}$, the matrix $U_{k}$ is (formally) of dimension $d \times d$. Since in image processing $d$ captures the number of pixels of one image, an explicit representation of $U_{k}$ consumes too much memory to be efficient which suggests to represent $U_{k}$ in terms of Householder reflections. This ensures that the memory consumption of the SVD of $A_{k}$ is bounded by $O\left(n_{k}^{2}+\right.$ $\left.r_{k} d\right)$, and the step $k+1$ requires $O\left(n_{k+1}^{3}+d m_{k}\left(r_{k}+m_{k}\right)\right)$ floating point operations.

\subsection{Thresholding-Adaptive SVD}

There already exist iterative methods to calculate an SVD, but for our purpose the approach from [23] has two favorable aspects. The first one is the possibility to perform blockwise updates with $m_{k}>1$, that is, with several frames. The second one is the ability to estimate the effect of appending $B_{k}$ on the singular values of $A_{k+1}$. In order to compute the SVD of $A_{k+1}, Z:=U_{k}^{T} B_{k}$ is first calculated and a $Q R$ decomposition with column pivoting of $Z_{r_{k}+1: d,:}=Q R P$ is determined. The $R$ matrix contains the information in the added data $B_{k}$ that is not already described by the singular vectors in $U_{k}$. Then, the matrix $R$ can be truncated by a significance level $\tau$ such that the singular values less than $\tau$ are set to zero in the SVD calculation of

$\left[\begin{array}{cc}\Sigma_{k}^{\prime} & Z_{1: r_{k},:} P^{T} \\ & R\end{array}\right]$

Therefore, one can determine only from the (cheap) calculation of a QR decomposition, whether the new data contain significant new information and the threshold level $\tau$ can control how big the gain has to be for a data vector to be added to the current SVD decomposition in an iterative step.

\section{Description of the Algorithm}

In this section, we give a detailed description of our algorithm to compute a background separation based on the adaptive SVD.

\subsection{Essential Functionalities}

The algorithm in [23] was initially designed with the goal to determine the kernels of a sequence of columnwise augmented matrices using the $V$ matrix of the SVDs. In background subtraction, on the other hand, we are interested in finding a low-rank approximation of the column space of $A$ and therefore concentrate on the $U$ matrix of the SVD which will allow us to avoid the computation and storage of $V$.

The adaptive SVD algorithm starts with an initialization step called SVDComp, calculating left singular vectors and singular values on an initial set of data. Afterward, data are added iteratively by blocks of arbitrary size. For every frame in such a block, the foreground is determined and then the SVDAppend step performs a thresholding described in Sect. 3.2 to check whether the frame is considered in the update of the singular vectors and values that corresponding to the background. 


\subsubsection{SVDComp}

SVDComp performs the initialization of the iterative algorithm. It is given the matrix $A \in \mathbb{R}^{d \times n}$ and a column number $\ell$ and computes the best rank- $\ell$ approximation $A=U \Sigma V^{T}$,

$U=:\left[U_{0}, U_{0}^{\prime}\right], \quad \Sigma=:\left[\begin{array}{cc}\Sigma_{0} & 0 \\ 0 & \Sigma_{0}^{\prime}\end{array}\right], \quad V=:\left[V_{0}, V_{0}^{\prime}\right]$,

by means of an SVD with $\Sigma_{0} \in \mathbb{R}^{\ell \times \ell}, U_{0} \in \mathbb{R}^{d \times \ell}$, and $V_{0} \in \mathbb{R}^{n \times \ell}$. Also, this SVD is conveniently computed by means of the algorithm from [23], as the thresholding of the augmented SVD will only compute and store an at most rank$\ell$ approximation, truncating the $R$ matrix in the augmentation step to at most $\ell$ columns. This holds both for initialization and update in the iterative SVD.

As mentioned already in Sect. 3.1, $U_{0}$ is not stored explicitly but in the form of Householder vectors $h_{j}, j=1, \ldots, \ell$, stored in a matrix $H_{0}$. Together with a small matrix $\widetilde{U}_{0} \in$ $\mathbb{R}^{\ell \times \ell}$, we then have

$U_{0}=\widetilde{U}_{0} \prod_{j=1}^{\ell}\left(I-h_{j} h_{j}^{T}\right)$,

and multiplication with $U_{0}$ is easily performed by doing $\ell$ Householder reflection and then multiplying with an $\ell \times \ell$ matrix. Since $V_{0}$ is not needed in the algorithm, it is neither computed nor stored.

\subsubsection{SVDAppend}

This core functionality augments a matrix $A_{k}$, given by $\widetilde{U}_{k}, \Sigma_{k}, H_{k}$, determined either by SVDComp or previous applications of SVDAppend, by $m$ new frames contained in the matrix $B \in \mathbb{R}^{d \times m}$ as described in Sect. 3.1. The details of this algorithm based on Householder representation can be found in [23]. By the thresholding procedure from Sect. 3.2, one can determine, even before the calculation of the SVD if an added column is significant relative to the threshold level $\tau$. This saves computational capacities by avoiding the expensive computation of the SVD for images that do not significantly change the singular vectors representing the background.

The choice of $\tau$ is significant for the performance of the algorithm. The basic assumption for the adaptive SVD is that the foreground consists of small changes between frames. Calculating SVDComp on an initial set of frames and considering the singular vectors, i.e., the columns of $U_{0}$, and the respective singular values give an estimate for the size of the singular values that correspond to singular vectors describing the background. With a priori knowledge of the maximal size of foreground effects, $\tau$ can even be set absolutely to the size of singular values that should be accepted. Of course, this approach requires domain knowledge and is not entirely data driven.

Another heuristic choice of $\tau$ can be made by considering the difference between two neighboring singular values $\sigma_{i}-\sigma_{i+1}$, i.e., the discrete slope of the singular values. The last and smallest singular values describe the least dominant effects. These model foreground effects or small, negligible effects in the background. With increasing singular values, the importance of the singular vectors is growing. Based on that intuition, one can set a threshold for the difference of two consecutive singular values and take the first singular value exceeding the difference threshold as $\tau$. Figure 1d illustrates a typical distribution of singular values. Since we want the method to be entirely data driven, we choose this approach. The threshold $\tau$ is determined by $\hat{i}:=\min \left\{i: \sigma_{i}-\sigma_{i+1}<\tau^{*}\right\}$ and $\tau=\sigma_{\hat{i}}$ with the threshold $\tau^{*}$ of the slope being determined in the following.

\subsubsection{Re-Initialization}

The memory footprint at the $k$ th step in the algorithm described in Sect. 3.1 is $O\left(n_{k}^{2}+r_{k} d\right)$ and grows with every frame added in the SVDAppend step. Therefore, a reinitialization of the decomposition is necessary.

One possibility is to compute an approximation of $A_{k} \approx$ $U_{k} \Sigma_{k} V_{k}^{T} \in \mathbb{R}^{d \times n_{k}}$ or the exact matrix $A_{k}$ by applying SVDComp to $A_{k}$ with a rank limit of $\ell$ that determines the number of singular vectors after re-initialization. This strategy has two disadvantages. The first one is that it needs $V_{k}$, which is otherwise not needed for modeling the background, and hence would require unnecessary computations. Even worse, though $\widetilde{U}_{0} \in \mathbb{R}^{\ell \times \ell}, \Sigma_{0} \in \mathbb{R}^{\ell \times \ell}$, and $H_{0} \in \mathbb{R}^{d \times \ell}$ are reduced properly, the memory consumption of $V_{0} \in \mathbb{R}^{n_{k} \times \ell}$ still depends on the number of frames added so far.

The second re-initialization strategy, referred to as (II), builds on the idea of a rank- $\ell$ approximation of a set of frames representing mostly the background. For every frame $B_{i}$ added in step $k$ of the SVDAppend, the orthogonal projection

$U_{k}(:, 1: \hat{i})\left(U_{k}(:, 1: \hat{i})^{T} B_{i}\right)$,

i.e., the "background part" of $B_{i}$, gets stored successively. The value $\sigma_{\hat{i}}$ is determined in Sect. 4.1.2 as threshold for the SVDAppend step. If the number of stored background images exceeds a fixed size $\mu$, the re-initialization gets performed via SVDComp on the background images. No matrix $V$ is necessary for this strategy, and the re-initialization is based on the background projection of the most recently appended frames.

In the final algorithm, we use a third strategy, referred to as (III) which is inspired by the sequential Karhunen-Loeve 


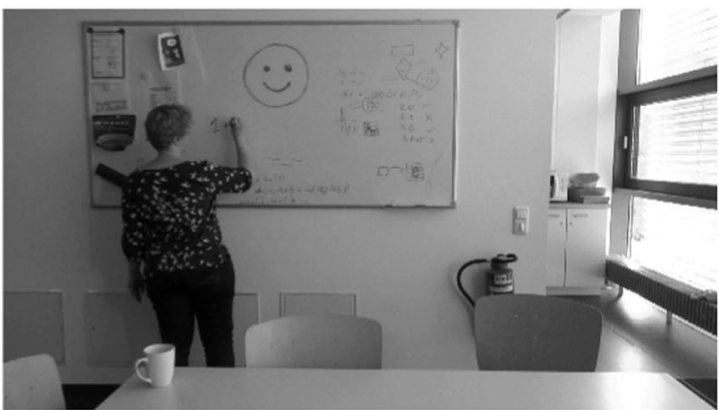

(a) Original image

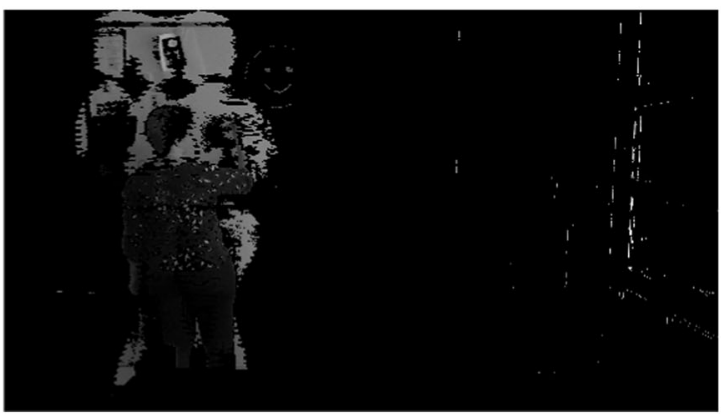

(c) Foreground image

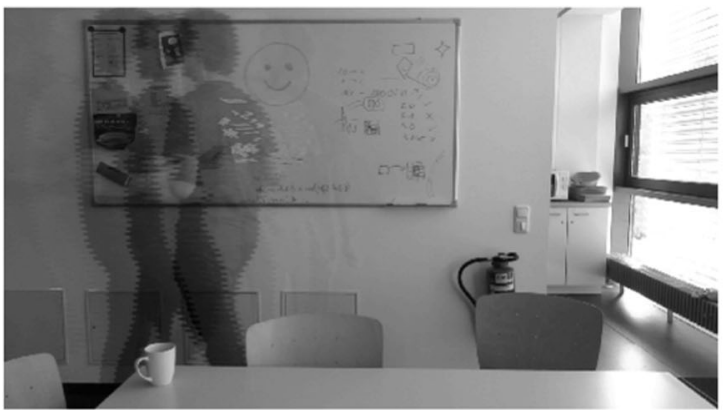

(b) Orthogonal projection onto background subspace

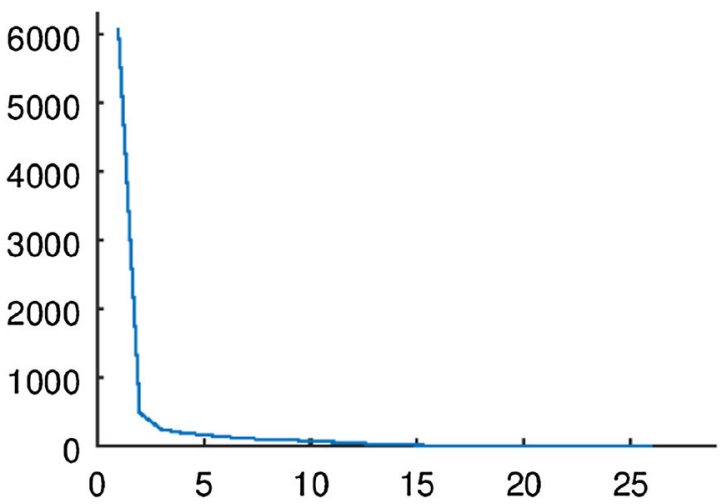

(d) Magnitude of the singular values plotted over the position on the diagonal of $\Sigma$

Fig. 1 Example of artifacts due to a big foreground object that was added to the background. The foreground object in the original image (a) triggers singular vectors containing foreground objects falsely added to the background (b) in previous steps. These artifacts can thus be seen in the foreground image (c)

basis extraction [16]. The setting is very similar, and the $V$ matrix gets dropped after the initialization as well. The update step with a data matrix $B_{k}$ is performed just like the update step of the iterative SVD calculation in Sect. 3.1 based on the matrix $\left[U_{k} \Sigma_{k}, B_{k}\right.$ ]. The matrices $\Sigma_{k+1}$ and $U_{k+1}$ get truncated by a thresholding of the singular values at every update step. Due to this thresholding, the number of singular values and accordingly the number of columns of $U_{k}$ have an upper bound. Therefore, the maximum size of the system is fixed and no re-initialization is necessary. Calculating the SVD of $\left[U_{k} \Sigma_{k}, B_{k}\right]$ is sufficient since due to

$$
\begin{aligned}
{\left[U_{k} \Sigma_{k}, B_{k}\right]\left[U_{k} \Sigma_{k}, B_{k}\right]^{T} } & =U_{k} \Sigma_{k} \Sigma_{k}^{T} U_{k}^{T}+B_{k} B_{k}^{T} \\
& =U_{k} \Sigma_{k} V_{k}^{T} V_{k} \Sigma_{k}^{T} U_{k}^{T}+B_{k} B_{k}^{T} \\
& =\left[U_{k} \Sigma_{k} V_{k}^{T}, B_{k}\right]\left[U_{k} \Sigma_{k} V_{k}^{T}, B_{k}\right]^{T}
\end{aligned}
$$

the eigenvectors and eigenvalues of the correlation matrices with respect to $\left[U_{k} \Sigma_{k}, B_{k}\right]$ and $\left[U_{k} \Sigma_{k} V_{k}^{T}, B_{k}\right]$ are the same. Therefore, the singular values of $\left[U_{k} \Sigma_{k}, B_{k}\right]$ and $\left[U_{k} \Sigma_{k} V_{k}^{T}, B_{k}\right]$ are the same, being roots of the eigenvalues of the correlation matrix. In our approach, we combine the adaptive SVD with the re-initialization based on $U_{k} \Sigma_{k}$, i.e., we perform SVDComp on $U_{k} \Sigma_{k}$, because we want to keep the thresholding of the adaptive SVD. This is essentially the same as an update step in Karhunen-Loeve setting with $B_{k}=0$ and a more rigorous thresholding or a simple truncation of $U_{k}$ and $\Sigma_{k}$. The thresholding strategy of the adaptive SVD Sect. 3.2 is still valid, as the $\mathrm{QR}$ decomposition with column pivoting sorts the columns of the matrix according to the $\ell_{2}$ norm and the columns of $U_{k} \Sigma_{k}$ are ordered by the singular values due to $\left\|U \Sigma_{:, i}\right\|_{2}=\sigma_{i} . U_{k} \Sigma_{k}$ already is in SVD form, and therefore, SVDComp at re-initialization is reduced to a $\mathrm{QR}$ decomposition to regain Householder vectors and a truncation of $U_{k}$ and $\Sigma_{k}$ which is less costly than performing a full SVD.

Since it requires the $V$ matrix, the first re-initialization strategy will not be considered in the following, where we will compare only strategies (II) and (III).

\subsubsection{Normalization}

The concept of re-initialization via a truncation of $U_{k}$ and $\Sigma_{k}$ either directly through SVDComp of $U_{k} \Sigma_{k}$ or in the Karhunen-Loeve setting with thresholding of the singular values still has a flaw: The absolute value of the singular values grows with each frame appended to $U_{k} \Sigma_{k}$ as 
$\sum_{i=1}^{n} \sigma_{i}^{2}=\|A\|_{F}^{2}$.

This also accounts for

$$
\begin{aligned}
\sum_{i=1}^{n_{k+1}} \sigma_{n_{k+1}, i}^{2} & =\left\|U_{k+1} \Sigma_{k+1}\right\|_{F}^{2} \\
& \approx\left\|\left[U_{k} \Sigma_{k}, B_{k}\right]\right\|_{F}^{2} \\
& =\left\|U_{k} \Sigma_{k}\right\|_{F}^{2}+\left\|B_{k}\right\|_{F}^{2} .
\end{aligned}
$$

The approximation results from the thresholding performed at the update step. As only small singular values get truncated, the sum of the squared singular values grows essentially with the Frobenius norm of the appended frames. Growing singular values do not only introduce numerical problems, they also deteriorate thresholding strategies, and the influence of newly added single frames decreases in later steps of the method. Therefore, some upper bound or normalization of the singular values is necessary.

Karhunen-Loeve [16] introduce a forgetting factor $\varphi \in$ $[0,1]$ and update as $\left[\varphi U_{k} \Sigma_{k}, B_{k}\right]$. They motivate this factor semantically: More recent frames get a higher weight. Ross et al. [26] show that this value limits the observation history. With an appending block size of $m$, the effective number of observations is $m /(1-\varphi)$. By the Frobenius norm argument, the singular values then have an upper bound. By the same motivation, the forgetting factor could also be integrated into strategy (III). Moreover, due to

$\left\|\left(\varphi U_{k} \Sigma_{k}\right)_{:, i}\right\|_{2}=\left\|\varphi \sigma_{i} U_{:, i}\right\|_{2}=\varphi \sigma_{i}$,

the multiplication with the forgetting factor keeps the order of the columns of $U_{k} \Sigma_{k}$ and linearly affects the 2-norm and is thus compliant with the thresholding. However, the concrete choice of the forgetting factor is unclear.

Another idea for normalization is to set an explicit upper bound for the Frobenius norm of observations contributing to the iterative SVD, or, equivalently, to $\sum \sigma_{i}^{2}=\|A\|_{F}^{2}$. At initialization, i.e., at the first SVDComp, the upper bound is determined by $\frac{\|A\|_{F}^{2}}{n} \eta$ with $n$ being the number of columns of $A$ and $\eta$ being the predefined maximum size of the system. This upper bound is a multiple of the mean squared Frobenius norm of an input frame, and we define a threshold $\rho:=$ $\frac{\|A\|_{F}}{\sqrt{n}} \sqrt{\eta}$. If the Frobenius norm $\left\|\Sigma_{0}\right\|_{F}$ of the singular values exceeds $\rho$ after a re-initialization step, $\Sigma_{0}$ gets normalized to $\Sigma_{0} \frac{\rho}{\left\|\Sigma_{0}\right\|_{F}}$. One advantage of this approach is that the effective system size can be transparently determined by the parameter $\eta$.

In data science, normalization usually aims for zero mean and standard deviation one. Zero mean over the pixels in the frames, however, leads to subtracting the rowwise mean of
$A$, replacing $A$ by $\left(I-11^{T}\right) A$. This approach is discussed in incremental PCA, cf. [26], but since the mean image usually contributes substantially to the background, it is not suitable in our application.

A framewise unit standard deviation makes sense since the standard deviation approximates the contrast in image processing and we are interested in the image content regardless of the often varying contrast of the individual frames. Different contrasts on a zero mean image can be seen as a scalar multiplication which also applies for the singular values. Singular values differing with respect to the contrast are not a desirable effect which is compensated by subtracting the mean and dividing by the standard deviation of incoming frames $B$, yielding $\frac{B-\mu}{\sigma}$. Due to the normalization of single images, the upper bound for the Frobenius norm $\rho$ is more a multiple of the Frobenius norm of an average image.

\subsection{Adaptive SVD Algorithm}

The essential components being described, we can now sketch our method based on the adaptive SVD in Algorithm 1.

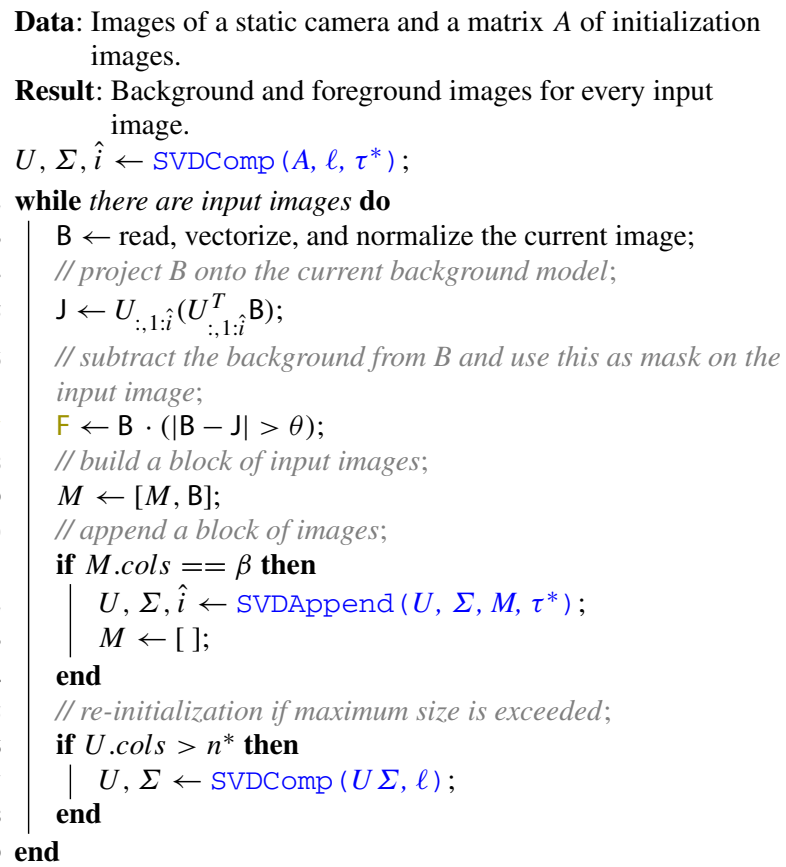

Algorithm 1: Background Subtraction using adaptive SVD.

The algorithm uses the following parameters:

- $\ell$ : Parameter used in SVDComp for rank- $\ell$ approximation.

- $\eta$ : Parameter for setting up the maximal Frobenius norm as a multiple of the Frobenius norm of an average image. 
- $\tau^{*}$ : Threshold value for the slope of the singular values used in SVDAppend.

- $\theta$ : Threshold value depending on the pixel intensity range to discard noise in the foreground image.

- $\beta$ : Number of frames put together to one block $B_{k}$ for SVDAppend.

- $n^{*}$ : Maximum number of columns of $U_{k}$. If $n^{*}$ is reached a re-initialization is triggered.

For the exposition in Algorithm 1, we use pseudo-code with a MATLAB like syntax. Two further explanations are necessary, however. First, we remark that SVDAppend and SVDComp return the updated matrices $U$ and $\Sigma$ and the index of the thresholding singular value determined by $\tau^{*}$ as described in Sect. 4.1.2. Using the threshold value $\theta$, the foreground resulting from the subtraction of the background from the input image gets binarized. This binarization is used as mask on the input image to gain the parts that are considered as foreground. $|B-J|>\theta$ checks elementwise whether $\left|B_{j k}-J_{j k}\right|>\theta$ and returns a matrix consisting of the Boolean values of this operation.

\subsection{Relaxation of the Small Foreground Assumption}

A basic assumption of our background subtracting algorithm is that the changes due to the foreground are small relative to the image size. Nevertheless, this assumption is easily violated, e.g., by a truck in traffic surveillance or generally by objects close to the camera which can appear in singular vectors that should represent background. This has two consequences. The first is that the foreground object is not recognized as such and the second one leads to ghosting effects because of the inner product as shown in Fig. 1.

The following modifications increase the robustness of our method against these unwanted effects.

\subsubsection{Similarity Check}

Big foreground objects can exceed the threshold level $\tau$ in SVDAppend and therefore are falsely included in the background space. With the additional assumption that background effects have to be stable over time, frames with large moving objects can be filtered out by utilizing the block appending property of the adaptive SVD. There, a large moving object causes significant differences in a block of images which can be detected by calculating the structural similarity of a block of new images. Wang et al. propose in [33] the normalized covariance of two images to capture the structural similarity. This again can be written as the inner product of normalized images, i.e.,

$s\left(B_{i}, B_{j}\right)=\frac{1}{d-1} \sum_{l=1}^{d} \frac{B_{i, l}-\mu_{i}}{\sigma_{i}} \frac{B_{j, l}-\mu_{j}}{\sigma_{j}}$,

with $B_{i}$ and $B_{j}$ being two vectorized images with $d$ pixels, mean values $\mu_{i}$ and $\mu_{j}$, and standard deviations $\sigma_{i}$ and $\sigma_{j}$. Considering that the input images already become normalized in our algorithm, see Sect. 4.1.4, this boils down to an inner product.

Given is a temporally equally spaced and ordered block of images $B:=\left\{B_{1}, B_{2}, \ldots, B_{m}\right\}$ and one frame $B_{i}$ with $i \in\{1,2, \ldots, m\}=: M$. The structural similarity of frame $B_{i}$ regarding the block $B$ is the measure we search for. This can be calculated by

$\frac{1}{m-1} \sum_{j \in M \backslash\{i\}} s\left(B_{i}, B_{j}\right)$

i.e., the mean structural similarity of $B_{i}$ regarding $B$. For the relatively short time span of one block, it generally holds that $s\left(B_{i}, B_{j}\right) \geq s\left(B_{i}, B_{k}\right)$ with $i, j, k \in M$ and $i<j<k$, i.e., the structural similarity drops going further into the future as motions in the images imply growing differences. This effect causes the mean structural similarity of the first or last frames of $B$ generally being lower than of the middle ones due to the higher mean time difference to the other frames in the block.

This bias can be avoided by calculating the mean similarity regarding subsets of $B$. Let $v>0$ be a fixed number of pairs to be considered for the calculation of the mean similarity and $\Delta T \in \mathbb{N}^{+}$be the fixed cumulative time difference. Calculate the mean similarity $\overline{s_{i}}$ of $B_{i}$ regarding $B$ by selecting pairwise distinct $\left\{j_{1}, j_{2}, \ldots, j_{v}\right\}$ from $M \backslash\{i\}$ with

$\sum_{l=1}^{v}\left|j_{l}-i\right|=\Delta T$ and $\overline{s_{i}}=\frac{1}{v}\left(\sum_{l=1}^{v} s\left(B_{i}, B_{j_{l}}\right)\right)$.

If $\overline{s_{i}}$ is smaller than the predefined similarity threshold $\bar{s}$, frame $i$ is not considered for the SVDAppend.

\subsubsection{Periodic Updates}

Using the threshold $\tau$ speeds up the iterative process, but also has a drawback: If the incoming images stay constant over a longer period of time, the background should mostly represent the input images and there should be high singular values associated with the singular vectors describing it. Since input images that can be explained well do not get appended anymore, this is, however, not the case. Another drawback is that outdated effects, like objects that stayed in the focus for quite some time and then left again, have 
a higher singular vectors than they should, as they are not relevant anymore. Therefore, it makes sense to periodically append images although they are seen as irrelevant and do not surpass $\tau$. This also helps to remove falsely added foreground objects much faster.

\subsubsection{Effects of the Re-Initialization Strategy}

The re-initialization strategy (II) based on the background images $U_{k}(:, 1: \hat{i})\left(U_{k}(:, 1: \hat{i})^{T} B_{i}\right)$ as described in Sect. 4.1.3 supports the removal of incorrectly added foreground objects. When such an object, say $X$, is gone from the scene, i.e., $B_{i}$ does not contain $X$ and $U_{k}(:, 1: \hat{i})\left(U_{k}(:, 1: \hat{i})^{T} B_{i}\right)$ does not contain it either because a singular vector not containing $X$ approximates $B_{i}$ much better. As $X$ was added to the background, there must be at least one column $j^{*}$ of $U_{k}$ containing $X$, i.e., $U_{k}\left(:, 1: j^{*}\right)^{T} X \gg 0$. As $U_{k}(:, 1: \hat{i})\left(U_{k}(:, 1: \hat{i})^{T} B_{i}\right)$ does not contain $X,\left(U_{k}(:, 1: \hat{i})^{T}\right.$ $\left.B_{i}\right)_{j^{*}}$ must be close to zero as otherwise the weighted addition of singular vectors $U_{k}(:, 1: \hat{i})\left(U_{k}(:, 1: \hat{i})^{T} B_{i}\right)$ cancels $X$ out. The re-initialization is thus based on images not containing $X$, and the new singular vectors also do not contain leftovers of $X$ anymore.

Finally, the parameter $\eta$ modifies the size of the maximum Frobenius norm used for normalization in re-initialization strategy (III) from Sect. 4.1.4. A smaller $\eta$ reduces the importance of the already determined singular vectors spanning the background space and increases the impact of newly appended images. If an object $X$ was falsely added, it gets removed more quickly if current frames not containing $X$ have a higher impact. A similar behavior like with re-initialization strategy (II) can be achieved. The disadvantage is that the background model changes quickly and does not capture long time effects that well. In the end, it depends on the application which strategy performs better.

\section{Computational Results}

The evaluation of our algorithm is done based on an implementation in the $\mathrm{C}^{+}$programming language using Armadillo [27] for linear algebra computations.

\subsection{Default Parameter Setting}

Algorithm 1 depends on parameters that are still to be specified. In the following, we will introduce a default parameter setting that works well in many different applications. The parameters could even be improved or optimized for a specific application using ground-truth data. Our aim here, however, is to show that the adaptive SVD algorithm is a very generic one and applicable almost "out of the box" for various situations. The chosen default parameters are as follows:

$$
\begin{aligned}
& -\ell=15, \\
& -n^{*}=30, \\
& -\eta=30, \\
& -\tau^{*}=0.05 \cdot \rho, \text { with } \rho=\frac{\|A\|_{F}}{\sqrt{n}} \sqrt{\eta} \text { of the initialization } \\
& \quad \text { matrix } A \in \mathbb{R}^{d \times n}, \\
& -\beta=6, v=3, \Delta T=6, \bar{s}=0.97, \\
& -\theta=1.0 .
\end{aligned}
$$

The parameter $\ell$ determines how many singular values and corresponding singular vectors are kept after re-initialization. Setting $\ell$ too low can cause a loss of background information. In our examples, 15 turned out to be sufficient not to lose information. The re-initialization is triggered when $n^{*}$ relevant singular values have been accumulated. Choosing that parameter too big reduces the performance, as the floating point operations per SVDAppend step depend cubically on the number of singular vectors and linearly on the number of singular vectors times the number of pixels, see Sect. 3.1. The system size $\eta$ controls the impact of newly appended frames, and a large value of $\eta$ favors a stable background. The threshold value $\tau^{*}$ for the discrete slope of singular values in the SVDAppend step depends on the data. The heuristic factor 0.05 proved to be effective to indicate that the curve of the singular values flattens out. The block size $\beta$ and the corresponding $v$ and $\Delta T$ depend on the frame rate of the input. The choice is such that it does not delay the update of the background space too much, which would be the effect of a large block size. Keeping it relatively small, we are able to evaluate the input regarding similarity and stable effects. Due to the normalization of the input images to zero mean and standard deviation one, the similarity threshold $\bar{s}$ and the binarization threshold $\theta$ are stable against different input types.

\subsection{Small Foreground Objects}

The first example video for a qualitative evaluation is from a webcam monitoring the city of Passau, Germany, from above. The foreground objects, e.g., cars, pedestrians, and boats, are small or even very small. The frame rate of 2 frames per minute is relatively low, and the image size is $640 \times$ $480 \mathrm{px}$. This situation allows for a straightforward application of the basic adaptive SVD algorithm without similarity check and regular updates. The remaining parameters are as in the default setting of Sect. 5.1. 


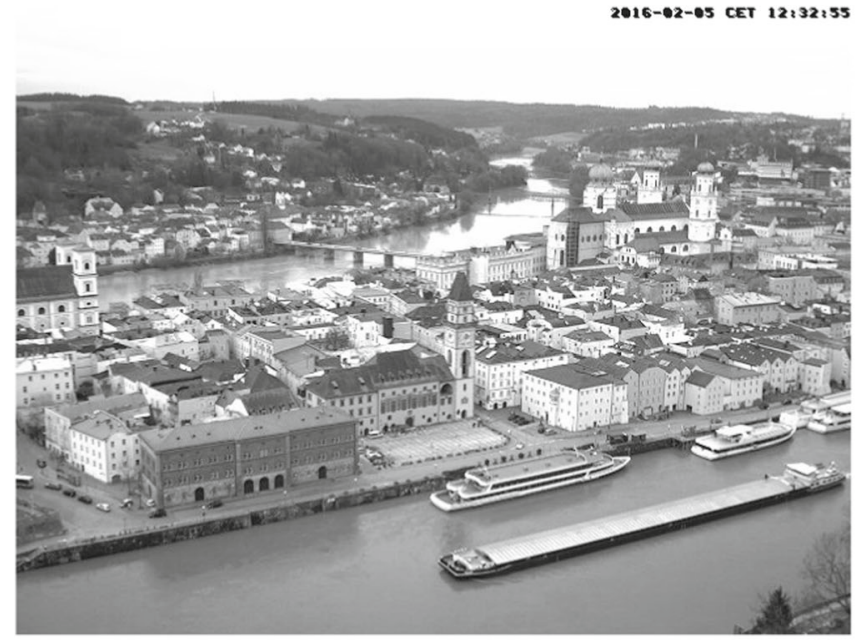

(a) Original image

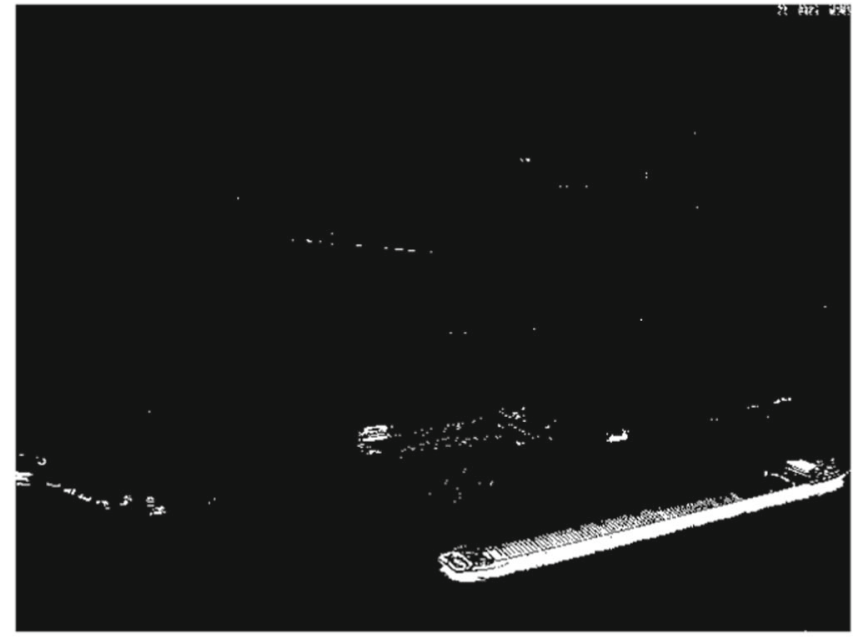

(b) Foreground image

Fig. 2 Example frame from a webcam video monitoring the city of Passau. In (a), the input image is shown and in (b) the foreground image as a result of Algorithm 1

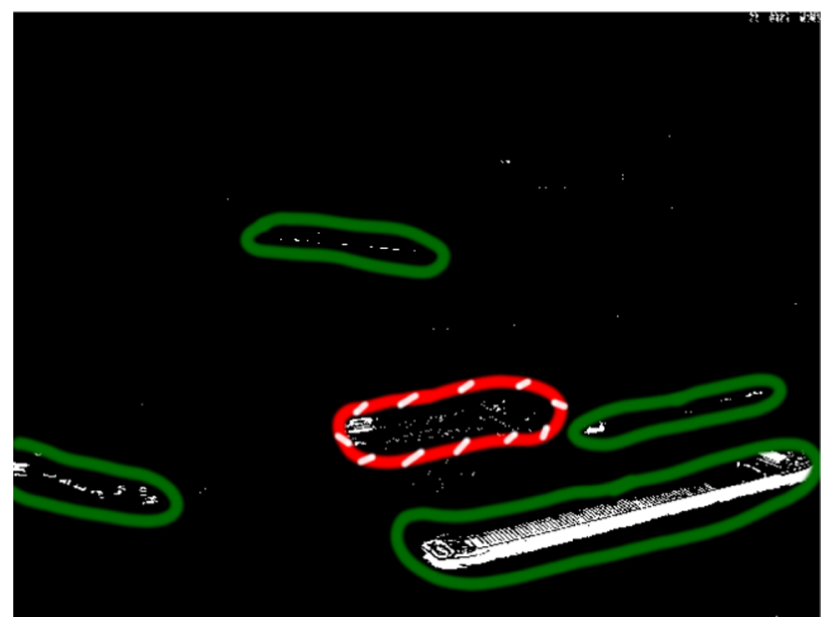

Fig. 3 Plot marking the true detections in the foreground image of Fig. $2 b$ by green circles and incorrect detections by red circles with white stripes (Color figure online)

In Fig. 2, an example frame ${ }^{1}$ and the according foreground image from the webcam video are shown. The moving boat in the foreground, the cars in the lower left and right corners, and even the cars on the bridge in the background are detected well. Small illumination changes and reparking vehicles lead to incorrect detections on the square in the front. Figure 3 depicts these regions.

\subsection{Handling of Big Foreground Objects}

Figure 1 is a frame from an example video ${ }^{1}$ including the projection onto the background space, the computed fore-

\footnotetext{
1 The complete sample videos can be downloaded following https:// www.forwiss.uni-passau.de/en/media_and_data/.
}

ground image, and the distribution of the singular values. To illustrate the improvements due to similarity checks and periodic updates, the same frame is depicted in Fig. 4 where the extended version of our algorithm is applied. The artifacts due to big foreground objects that were added to the background in previous frames are not visible anymore. The person in the image still gets added to the background, but only after being stationary for some frames.

\subsection{Execution Time}

The performance of our implementation is evaluated based on an Intel® Core ${ }^{\mathrm{TM}}$ i7-4790 CPU @ $3.60 \mathrm{~Hz} \times 8$. The example video from Sect. 5.3 has a resolution of $1920 \times$ 1080 px with $25 \mathrm{fps}$. For the application of our algorithm on the example video, the parameters are set as shown in Sect. 5.1.

As the video data were recorded with $25 \mathrm{fps}$, there is no need to consider every frame for a background update because the background is assumed to be constant over a series of frames and can only be detected considering a series of frames. Therefore, only every second frame is considered for a background update, while a background subtraction using the current singular vectors is performed on every frame. Our implementation with the settings from Sect. 5.1 handles this example video with 8 fps.

For surveillance applications, it is important that the background subtraction is applicable in real time for which $8 \mathrm{fps}$ are too slow. One approach would be to reduce the resolution. The effects of that will be discussed in the following section. Leaving the resolution unchanged, the parameters have to be adapted. Setting $\ell=10$ and $n^{*}=25$ signifi- 


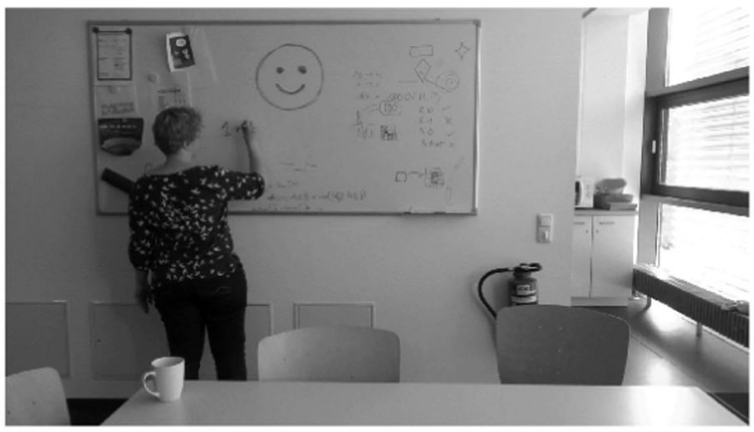

(a) Original image

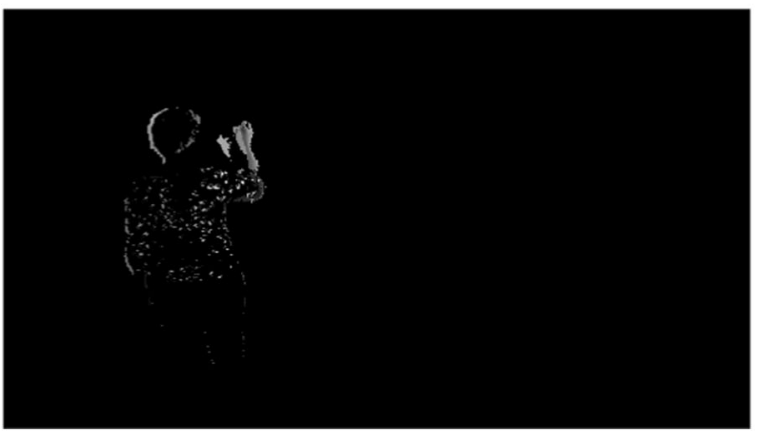

(c) Foreground image

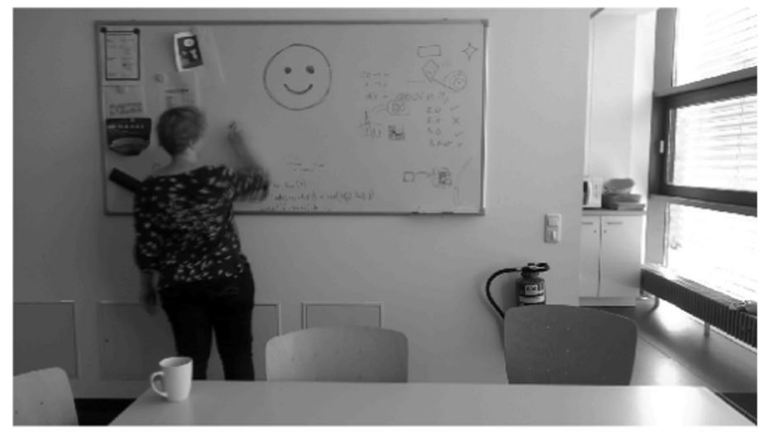

(b) Orthogonal projection onto background subspace

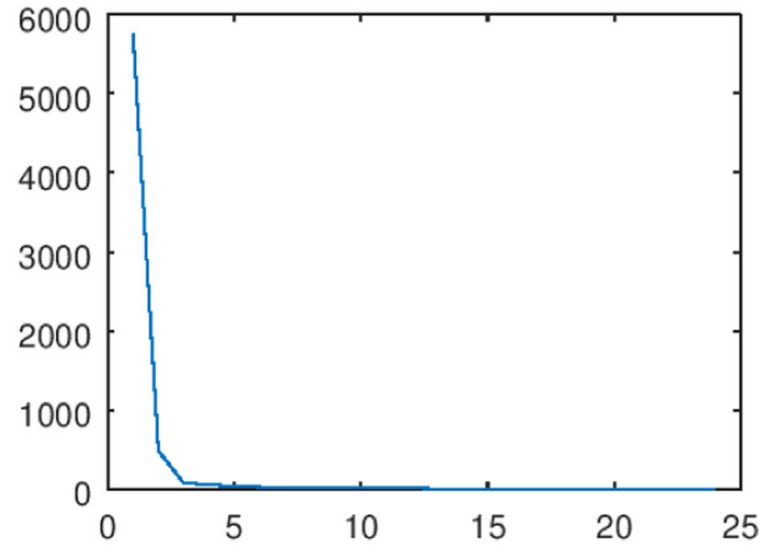

(d) Magnitude of the singular values plotted over the position on the diagonal of $\Sigma$

Fig. 4 The same scene as in Fig. 1. The artifacts due to big foreground objects are reduced by similarity checks and regular updates. The current foreground object gets added to the background only after being stationary for a series of frames

cantly reduces the number of background effects that can be captured, but turns out to be still sufficient for this particular scene. The number of images considered for background updates can be reduced as well. Downsampling the frames by averaging over a window size of 8 and setting $\ell=10$ and $n^{*}=25$ leads to a processing rate of $25 \mathrm{fps}$ which is real time.

In Sect. 3.1, we point out that the number of floating point operations for an update step depends linearly on the number of pixels $d$ when using Householder reflections. A reinitialization step is computationally even cheaper, because only Householder vectors have to be updated. The following execution time measurements underline the theoretical considerations. Our example video is resized several times, 900 images are appended, and re-initialization is performed when $n^{*}$ singular vectors are reached. Table 1 shows the summed up time for the append and re-initialization steps during iteration for the given image sizes. The number $d$ of pixels equals $2,073,600=1920 \cdot 1080$.

The factors $t_{d / i} /\left(t_{d / 16} \cdot \frac{16}{i}\right)$ with $i \in\{1,2,4,8,16\}$ and total append or re-initialization times $t_{d / i}$ are shown in Table 1 for image sizes $d / i$. These factors should be con-
Table 1 Execution time for performing an SVD update iteratively on 900 frames for different image sizes and $d=2073600=1920 \cdot 1080$

\begin{tabular}{lllll}
\hline \#Pixels & Append (s) & Factor & Re-init. (s) & Factor \\
\hline$d$ & 69.30 & 1.90 & 16.46 & 1.37 \\
$d / 2$ & 34.28 & 1.88 & 8.25 & 1.38 \\
$d / 4$ & 16.67 & 1.83 & 3.79 & 1.26 \\
$d / 8$ & 6.72 & 1.47 & 1.68 & 1.12 \\
$d / 16$ & 2.28 & 1 & 0.75 & 1 \\
\hline
\end{tabular}

stant for increasing image sizes due to the linear dependency. Still, the factors keep increasing, but even less than a logarithmic order. This additional increase in execution time can be explained due to the growing amount of memory that has to be managed and caching becomes less efficient as with small images.

\subsection{Evaluation on Benchmark Datasets}

The quantitative evaluation is performed on example videos from the background subtraction benchmark dataset CDnet 


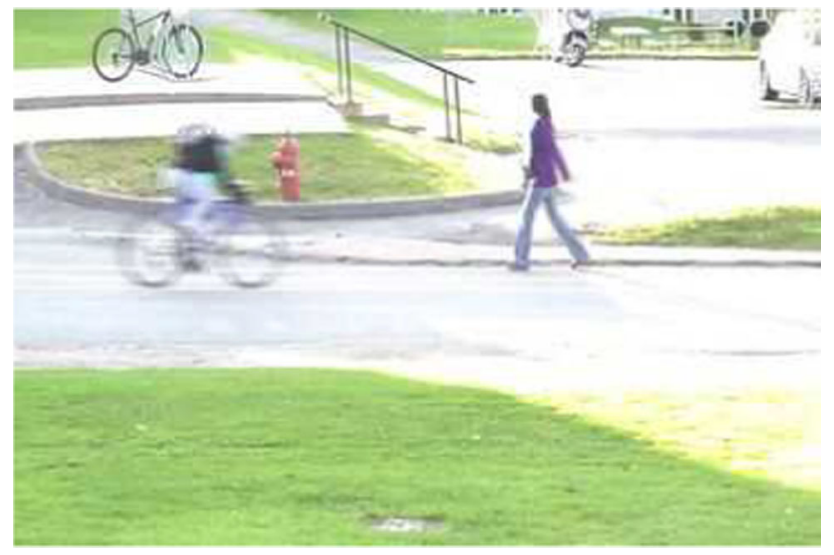

Fig. 5 Example frame from the pedestrians video of the CDnet database

2014 [32]. The first one is the pedestrians video belonging to the baseline category. It contains 1099 frames $(360 \times 240$ px) of people walking and cycling in the public. An example frame is shown in Fig. 5.

For the frames 300 through 1099, binary ground-truth annotations exist that distinguish between foreground and background. From the first 299 frames, 15 frames are equidistantly subsampled and taken for the initial matrix $M$. Thereafter, Algorithm 1 is executed on all frames from 300 through 1099. Instead of applying the binary mask in line 7 of Algorithm 1 onto the input image, the mask itself is the output to achieve binary images.

With the default parameter setting of Sect. 5.1, the pixelwise precision of 0.967 and F-Measure of 0.915 are achieved with a performance of $843 \mathrm{fps}$. The thresholding leading to the binary mask is sensitive to the contrast of the foreground relative to the background. If it is low, foreground pixels are not detected properly. To avoid missing pixels within foreground objects, the morphological close operation is performed with a circular kernel. Moreover, a fixed minimal size of foreground objects can be assumed reducing the number of false positives. These two optimizations lead to the precision of 0.973 and F-Measure of 0.954 at $684 \mathrm{fps}$. The complete evaluation measures are given in Table 2. Default represents the default parameter setting and morph the version with the additional optimizations. In the following, the morphological postprocessing is always included.
Table 3 Evaluation of the adaptive SVD algorithm on example videos from the CDnet dataset using precision and F-Measure. Prec* and F-Meas* give the precision and F-Measure of the best unsupervised method of the benchmark regarding the test video

\begin{tabular}{lllll}
\hline Video & Prec & F-Meas & Prec* & F-Meas* \\
\hline highway & 0.901 & 0.816 & 0.935 & 0.954 \\
park & 0.841 & 0.701 & 0.776 & 0.765 \\
tram & 0.957 & 0.812 & 0.838 & 0.886 \\
turnpike & 0.962 & 0.860 & 0.980 & 0.881 \\
blizzard & 0.919 & 0.854 & 0.939 & 0.845 \\
streetLight & 0.992 & 0.982 & 0.984 & 0.983 \\
\hline
\end{tabular}

Our method delivers a state-of-the-art performance for unsupervised methods. The best unsupervised method, IUTIS5 [1] on the benchmark site could achieve the precision of 0.955 and F-Measure of 0.969 . It is based on genetic programming combining other state-of-the-art algorithms. The execution time is not given, but naturally higher than the execution time of the slowest algorithm used, assuming perfectly parallel execution. We introduced domain knowledge only in the morphological optimizations. Otherwise, there is no specific change toward the test scene. Even more domain knowledge is used in supervised learning techniques as object shapes are trained and irrelevant movements in the background are excluded due to labeling. They are able to outperform our approach regarding the evaluation measures. An overall average precision and F-Measure of more than 0.98 is, for example, achieved from FgSegNet [17]. Nevertheless, the authors mention that their postprocessing is done on benchmark data leading to a bias as the short sequences look very much alike. Moreover, the benchmark site itself disclaims that the supervised methods may have been trained on evaluation data as ground-truth annotations are only available for evaluation data.

The positive effect of a blockwise appending of the data with a similarity check and regular updates as shown above also applies here: Our adaptive SVD algorithm on the given pedestrians video from the benchmark site without using the similarity checks and regular updates only leads to the precision of 0.933 and F-Measure of 0.931.

The performance of our algorithm on more example videos from the CDnet dataset is listed in Table 3. The park video is recorded with a thermal camera, the tram (CDnet:

Table 2 Evaluation of the pedestrians scene of the CDnet database with the benchmark evaluation metrics including FPR (false-positive rate), FNR (false-negative rate), PBC (percentage of wrong classifications)

\begin{tabular}{llllllll}
\hline & Recall & Specificity & FPR & FNR & PBC & Precision & F-Measure \\
\hline default & 0.869 & 1.000 & 0.000 & 0.131 & 0.158 & 0.967 & 0.915 \\
morph & 0.936 & 1.000 & 0.000 & 0.063 & 0.088 & 0.973 & 0.954 \\
\hline
\end{tabular}


tramCrossroad_1fps) and turnpike (CDnet: turnpike_0_5fps) videos with a low frame rate, and the blizzard video while snow is falling. For highway and park, the best unsupervised method is IUTIS-5 and for tram, turnpike, blizzard, and streetLight that is SemanticBGS [7]. SemanticBGS combines IUTIS-5 and a semantic segmentation deep neural network, and the execution time is given with $7 \mathrm{fps}$ for $473 \times 473$ px images based on a NVIDIA GeForce GTX Titan X GPU.

Besides the park video, the content is mostly vehicles driving by. The performance of our algorithm clearly drops whenever the initialization image set contains a lot of foreground objects like in the highway video, where the street is never empty. Moreover, a foreground object turns into background when it stops moving which is even a feature of our algorithm. This, however, causes problems in a lot of the benchmark videos of the CDnet dataset with vehicles stopping at traffic lights, like in the tram video, or people stopping and starting to move again. There is a category of videos with intermittent object motion in the CDnet benchmark. Our algorithm performs with an average precision of 0.752 and F-Measure of 0.385, whereas SemanticBGS reaches an average precision of 0.915 and F-Measure of 0.788 . The precision of our algorithm tends to be higher than the F-Measure, as it detects motion very well and therefore is certain that if there is movement, it is foreground, but often foreground is not detected due to a lack of motion. To delay the addition of a static object to the background, it is possible to reduce the regular updates, for example. But as this feature regulates the adaption of the background model to a change in the background, this only enhances the performance for very stable scenes. In the streetLight video, no regular update was performed in contrast to the other videos. Including regular updates, the precision is 0.959 and the F-Measure 0.622 due to cars stopping at traffic lights. The only domain knowledge we introduce is the postprocessing via morphological operations. Otherwise, the algorithm has no knowledge about the kind of background it models. Therefore, not only vehicles or people are detected as foreground, but also movement of trees or the reflection of the light of the vehicles on the ground, which is negative for the performance regarding the CDnet benchmark.

\section{Conclusions}

We utilized the iterative calculation of a singular value decomposition to model a common subspace of a series of frames which is assumed to represent the background of the frames. An algorithm, the adaptive SVD was developed and applied for background subtraction in image processing. The assumption that the foreground has to be small objects was considered in more detail and relaxed by extensions of the algorithm. In an extensive evaluation, the capabilities of our algorithm were shown qualitatively and quantitatively using example videos and benchmark results. Compared to state-of-the-art unsupervised methods, we obtain competitive performance with even superior execution time. Even high-definition videos can be processed in real time.

The evaluation also showed that if an application to a domain such as video surveillance is intended, our algorithm would need to be extended to also consider semantic information. Therefore, it can only be seen as a preprocessing step, e.g., reducing the search space for classification algorithms. In future work, we aim to evaluate the benefit of using our algorithm in preprocessing of an object classifier. Moreover, we will address the issue of foreground objects turning into background after being static for some time which is desirable in some cases and erroneous in others. A first approach is to use tracking because objects do not disappear without any movement. In the end, there is also some parallelization ability in our algorithm separating the projection onto the background of incoming images from the update of the background model. Further performance improvements will be investigated.

Acknowledgements Open Access funding provided by Projekt DEAL. Our work results from the project DeCoInt ${ }^{2}$, supported by the German Research Foundation (DFG) within the priority program SPP 1835: "Kooperativ interagierende Automobile," Grant Numbers DO 1186/11, FU 1005/1-1, and SI 674/11-1.

Open Access This article is licensed under a Creative Commons Attribution 4.0 International License, which permits use, sharing, adaptation, distribution and reproduction in any medium or format, as long as you give appropriate credit to the original author(s) and the source, provide a link to the Creative Commons licence, and indicate if changes were made. The images or other third party material in this article are included in the article's Creative Commons licence, unless indicated otherwise in a credit line to the material. If material is not included in the article's Creative Commons licence and your intended use is not permitted by statutory regulation or exceeds the permitted use, you will need to obtain permission directly from the copyright holder. To view a copy of this licence, visit http://creativecomm ons.org/licenses/by/4.0/.

\section{References}

1. Bianco, S., Ciocca, G., Schettini, R.: Combination of video change detection algorithms by genetic programming. IEEE Trans. Evolut. Comput. 21(6), 914-928 (2017)

2. Bouwmans, T.: Recent advanced statistical background modeling for foreground detection: a systematic survey. Recent Patents Comput. Sci. 4, 147-176 (2011)

3. Bouwmans, T.: Traditional and recent approaches in background modeling for foreground detection: an overview. Comput. Sci. Rev. 11-12, 31-66 (2014)

4. Bouwmans, T., Javed, S., Sultana, M., Jung, S.K.: Deep neural network concepts for background subtraction: a systematic review and comparative evaluation. Neural Netw. 117, 8-66 (2019) 
5. Bouwmans, T., Sobral, A., Javed, S., Jung, S.K., Zahzah, E.H.: Decomposition into low-rank plus additive matrices for background/foreground separation: a review for a comparative evaluation with a large-scale dataset. Comput. Sci. Rev. 23, 1-71 (2017)

6. Bouwmans, T., Zahzah, E.H.: Robust PCA via principal component pursuit: a review for a comparative evaluation in video surveillance. Comput. Vis. Image Understand. 122, 22-34 (2014)

7. Braham, M., Piérard, S., Van Droogenbroeck, M.: Semantic background subtraction. In: 2017 IEEE International Conference on Image Processing (ICIP), pp. 4552-4556 (2017)

8. Candès, E.J., Li, X., Ma, Y., Wright, J.: Robust principal component analysis? J. ACM 58(3), 1-37 (2011)

9. Erichson, N.B., Brunton, S.L., Kutz, J.N.: Compressed singular value decomposition for image and video processing. In: 2017 IEEE International Conference on Computer Vision Workshops (ICCVW), pp. 1880-1888 (2017)

10. Golub, G.H., Van Loan, C.F.: Matrix Computations, 3rd edn. Johns Hopkins University Press, Baltimore (1996)

11. Goodfellow, I., Bengio, Y., Courville, A.: Deep Learning. MIT Press (2016). http://www.deeplearningbook.org

12. Gracewell, J., John, M.: Dynamic background modeling using deep learning autoencoder network. Multimed. Tools Appl. 79(7), 46394659 (2020)

13. Guo, H., Qiu, C., Vaswani, N.: Practical ReProCS for separating sparse and low-dimensional signal sequences from their sum-part 1. In: 2014 IEEE International Conference on Acoustics, Speech and Signal Processing (ICASSP), pp. 4161-4165 (2014)

14. Kaloorazi, M.F., de Lamare, R.C.: Randomized rank-revealing UZV decomposition for low-rank approximation of matrices (2018). arXiv: 1811.08597

15. LeCun, Y., Bottou, L., Bengio, Y., Haffner, P.: Gradient-based learning applied to document recognition. Proc. IEEE 86, 22782324 (1998)

16. Levey, A., Lindenbaum, M.: Sequential Karhunen-Loeve basis extraction and its application to images. IEEE Trans. Image Process. 9(8), 1371-1374 (2000)

17. Lim, L.A., Keles, H.Y.: Foreground segmentation using convolutional neural networks for multiscale feature encoding. Pattern Recognit Lett. 112, 256-262 (2018)

18. Liu, X., Wen, Z., Zhang, Y.: Limited memory block Krylov subspace optimization for computing dominant singular value decompositions. SIAM J. Sci. Comput. 35(3), A1641-A1668 (2013)

19. Lu, Y., Ino, F., Matsushita, Y.: High-performance out-of-core block randomized singular value decomposition on GPU (2017). arXiv:1706.07191

20. Minematsu, T., Shimada, A., Uchiyama, H., Taniguchi, R.: Analytics of deep neural network-based background subtraction. MDPI J. Imag. 4(6), 78 (2018)

21. Mnih, V., Kavukcuoglu, K., Silver, D., Rusu, A.A., Veness, J., Bellemare, M.G., Graves, A., Riedmiller, M., Fidjeland, A.K., Ostrovski, G., Petersen, S., Beattie, C., Sadik, A., Antonoglou, I., King, H., Kumaran, D., Wierstra, D., Legg, S., Hassabis, D.: Human-level control through deep reinforcement learning. Nature pp. 518-529 (2015)

22. Narayanamurthy, P., Vaswani, N.: A fast and memory-efficient algorithm for robust PCA (MEROP). In: 2018 IEEE International Conference on Acoustics, Speech and Signal Processing (ICASSP), pp. 4684-4688 (2018)

23. Peña, J.M., Sauer, T.: SVD update methods for large matrices and applications. Linear Algebra Appl. 561, 41-62 (2019)

24. Rodriguez, P., Wohlberg, B.: Incremental principal component pursuit for video background modeling. J. Math. Imag. Vis. 55(1), 1-18 (2016)

25. Rodríguez, P., Wohlberg, B.: Translational and rotational jitter invariant incremental principal component pursuit for video back- ground modeling. In: 2015 IEEE International Conference on Image Processing (ICIP), pp. 537-541 (2015)

26. Ross, D.A., Lim, J., Lin, R.S., Yang, M.H.: Incremental learning for robust visual tracking. Int. J. Comput. Vis. 77(1), 125-141 (2008)

27. Sanderson, C., Curtin, R.: Armadillo: a template-based C++ library for linear algebra. J. Open Source Softw. 1, 26 (2016)

28. Schmidt, E.: Zur Theorie der linearen und nichtlinearen Integralgleichungen. I. Teil. Entwicklung willkürlicher Funktionen nach Systemen vorgeschriebener. Math. Annalen 63, 433-476 (1907)

29. Stewart, G.W.: On the early history of the singular value decomposition. SIAM Rev. 35(4), 551-566 (1993)

30. Sultana, M., Mahmood, A., Javed, S., Jung, S.K.: Unsupervised deep context prediction for background estimation and foreground segmentation. Multimed. Tools Appl. 30(3), 375-395 (2019)

31. Vaswani, N., Bouwmans, T., Javed, S., Narayanamurthy, P.: Robust subspace learning: robust PCA, robust subspace tracking, and robust subspace recovery. IEEE Signal Process. Mag. 35(4), 32-55 (2018)

32. Wang, Y., Jodoin, P., Porikli, F., Konrad, J., Benezeth, Y., Ishwar, P.: CDnet 2014: an expanded change detection benchmark dataset. In: 2014 IEEE Conference on Computer Vision and Pattern Recognition Workshops, pp. 393-400 (2014)

33. Wang, Z., Bovik, A.C., Sheikh, H.R., Simoncelli, E.P.: Image quality assessment: from error visibility to structural similarity. IEEE Trans. Image Process. 13(4), 600-612 (2004)

Publisher's Note Springer Nature remains neutral with regard to jurisdictional claims in published maps and institutional affiliations.

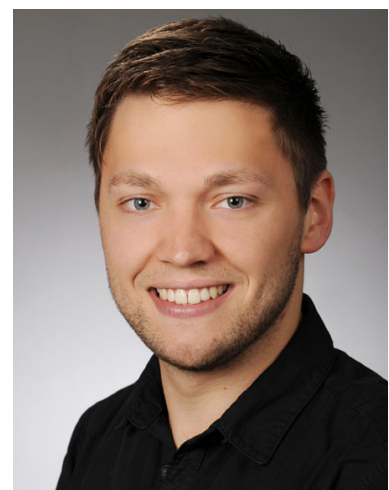

Günther Reitberger received the B.Sc. and the M.Sc. degrees in Computer Sciences from the University of Passau, Germany, in 2014 and 2016, respectively. Currently, he is working on his $\mathrm{PhD}$ at the Institute for Software Systems in Technical Applications of Computer Science (FORWISS) of the University of Passau, Germany. His research interests include mathematical image processing, stereo vision, artificial intelligence, object detection, and object tracking.

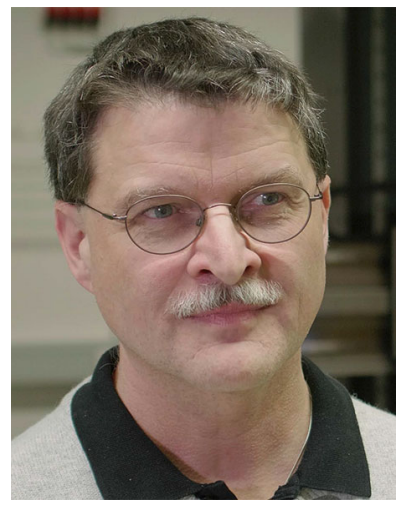

Tomas Sauer received his $\mathrm{PhD}$ degree in mathematics from the University of Erlangen-Nürnberg in 1993 for research in Approximation Theory. From 2000 to 2012, he was professor for Numerical Mathematics/Scientific Computation at the University of Gießen, and since 2012, he holds the chair for Mathematical Image Processing at the University of Passau and is also director of the Applied Research Institute FORWISS and of the Fraunhofer IIS Research Group on "Knowledge-Based Image Processing." His current research interests include, among others, signal and image processing for huge data, tomography, machine learning and sparse reconstructions, and continued fractions. 